\title{
EFICIÊNCIA SIMBIÓTICA DE FUNGOS MICORRÍZICOS ARBUSCULARES EM SOLO NÃO FUMIGADO, PARA MAMOEIRO(1)
}

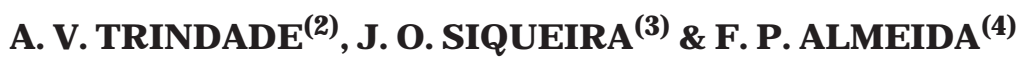

\begin{abstract}
RESUMO
Para que os fungos micorrízicos arbusculares (FMA) possam ser utilizados em um programa de inoculação, é necessário que sejam capazes de apresentar eficiência simbiótica em solo que contenha populações indígenas de FMA. Com o objetivo de avaliar a eficiência si mbiótica e o potencial de inoculação de fungos MA em solo não fumigado, para o mamoeiro, foi desenvolvido um experimento em condições de casa de vegetação da E mbrapa Mandioca e Fruticultura, Cruz das Almas (BA), utilizando a variedade de mamoeiro Tainung $\mathbf{n}^{\circ} \mathbf{1}$. Utilizou-se

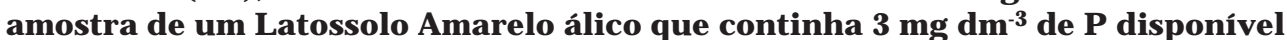
e que recebeu doses crescentes de $P\left(0,20,40,80\right.$ e $\left.140 \mathrm{mg} \mathrm{dm}^{-3}\right)$, combinadas com inoculação de três espécies previamente selecionadas e três isolados nativos de FMA, obtidos de agrossistema de mamoei ro. As plantas foram inoculadas com solo-inóculo no ato da repicagem e cultivadas por 50 dias, quando se determinaram a colonização, matéria seca da parte aérea e teores de nutrientes nas plantas. Todos os fungos inoculados apresentaram eficiência si mbiótica em solo não fumigado, destacando-se Glomus clarum, Gi gaspora margarita e isolado 29 (Gigaspora sp.), que apresentaram eficiência alta. Os isolados nativos foram mais eficientes em doses mais elevadas de fósforo no solo; a eficiência esteve relacionada com a absorção de fósforo e potássio. Os fungos previamente selecionados em solo fumigado foram também eficientes em solo que continha população indígena de FMA, portanto, validando este procedimento.
\end{abstract}

Palavras-chave: Carica papaya, mamão, fumigação, inoculação, micorriza, Gigaspora, Glomus, fungos indígenas.

\footnotetext{
(1) Trabalho extraído de Tese de Doutorado do primeiro autor, apresentada ao curso de Solos e Nutrição de PIantas do DCS/UFLA, Lavras (MG). Recebido para publicação em setembro de 1999 e aprovado em junho de 2000.

(2) Pesquisador da Embrapa Mandioca e Fruticultura. Rua Embrapa s/n, Caixa Postal 007, CEP 44380-000 Cruz das Almas (BA). Email: aldo@cnpmf.embrapa.br

(3) Professor da Universidade Federal de Lavras - UFLA, Caixa Postal 37, CEP 37200-000 Lavras (MG).

(4) Engenheiro-Agrônomo, Universidade Federal da Paraíba, Pós-graduação, CEP 58102-970 Campina Grande (PB).
} 


\title{
SUMMARY: SYMBIOTICEFFICIENCY OF ARBUSCULAR MYCORRHIZAL IN UNFUMIGATED SOIL FUNGI FOR PAPAYA
}

\begin{abstract}
In order to beused in a program of inoculation, efficient arbuscular mycorrhizal fungi (AMF) must be able to cope with the indigeneous fungal population. An experiment was conducted under greenhouse conditions at the Embrapa Cassava and Fruit Crops in Cruz das Almas (BA), Brazil, with the objective to evaluate the competitive capability of selected fungi, using natural soil with Tainung no. 1 variety. A Yellow Oxisol with $3 \mathrm{mg} \mathrm{dm}^{-3}$ of availableP was used. Three exotic and three native fungi were tested in the presence of five $P$ levels $\left(0,20,40,80\right.$ and $\left.140 \mathrm{mg} \mathrm{dm}^{-3}\right)$. Plants werecul tured for 50 days after inoculation/ transplanting and shoot dry weigth, nutrient contents and total percent col onization were determined. All inoculated fungi were more effective than the indigenous fungi, especially G. clarum, G. margarita and isol ate29; nativeisol ates weremoreeffectivein higher levels of $P$; effectiveness was related to $P$ and $K$ absorption. Ther efore, thefungi initially selected in fumigated soil were equally effective in unfumigated soil, under competitive conditions.

Index terms: Carica papaya, fumigation, inoculation, mycorrhiza, Gigaspora, Glomus, indigenous fungi.
\end{abstract}

\section{INTRODUÇÃO}

Os fungos micorrízicos arbusculares (F MAs) têm sido estudados, visando à sua aplicação para incrementar o desenvolvimento e a produção das culturas mediante seus efeitos na nutrição das plantas e outros benefícios diretos e indiretos. Entretanto, existem al guns obstácul os a isto, dentre os quais a adaptação aos fatores edáficos e competição com fungos indígenas, que podem comprometer a eficiência simbiótica dos FMAs introduzi dos na inoculação (M anjunath et al ., 1983; Balakrishna et al., 1996).

Em sentido restrito, a eficiência simbiótica dos fungos MA, resulta de interações complexas entrea capacidade da planta em satisfazer seus requerimentos de $\mathrm{P}$ e a habilidade do fungo em prover esse nutriente à planta hospedeira (Koide, 1991). Em sentido mais amplo, fungo eficiente é aquele que, em dadas condições defertilidade do solo, consegue sobreviver, colonizar as raízes, competir com outros microrganismos, incluindo outros FMAs, produzir grande volume de micélio e estabelecer relação mutual ista eficiente com a planta (Siqueira, 1991). Quando os fungos são aval iados em condições diferentes defertilidade, pode-se estimar a eficiência de cada fungo em relação à população nativa (Saggin Júnior \& Siqueira, 1995). Por meio desse procedimento pode-se prever o grau de sucesso ou fracasso da inoculação em condições de campo em solos com diferentes caraterísticas químicas e populações de FMAs indígenas.

Dentre os fatores que definem a infectividadee a capacidade competitiva de um fungo, destacam-se: o tipo, a viabilidade e o número de seus propágulos, os quais determinam o potencial infectivo ou de inóculo do fungo ou população. Powell et al. (1980) relataram uma relação inversa entre a densidade de inóculo nativo no sol o e a resposta de crescimento da planta à inoculação, demonstrando a importância do potencial de inóculo nativo sobre o sucesso da inoculação. Entretanto, mesmo em solos com elevado potencial de inóculo, a inoculação com fungos eficientes pode gerar respostas positivas na planta (Medina et al., 1988), indicando que, em adição à abundância de fungos indígenas, informações sobre sua infectividade e eficiência sob condições de solo adubado são necessárias para avaliar a potencialidade dos locais para responsividade à inoculação com F MA efetivo. Conhecer a quantidade e qualidade dos FMAs indígenas e a eficiência do fungo a ser introduzido são pré-requisitos para o sucesso de uma inoculação em campo (Wilson, 1984).

O mamoeiro é uma planta com el evada resposta à colonização micorrízica (Silva \& Siqueira, 1991; Weber \& Amorim, 1994). Isolados das espécies Gigaspora cal ospora (Ramirez et al., 1975), Gl omus clarum (Silva \& Siqueira, 1991), G. macrocarpum, (Mohandas, 1992; Auler, 1995), G. mosseae (Mohandas, 1992; Balakrishna et al., 1996) e G. etunicatum (Weber \& Amorim, 1994) têm mostrado boas respostas quando inoculados nesta cultura. Entretanto, todos estes estudos empregaram solos desinfestados. Apenas Balakrishna et al. (1996) realizarama seleção deFMA em solonão desinfestado, tendo comprovado a eficiência de G. mosseae em condições de competição.

Este trabalho objetivou avaliar, em solo não fumigado, a eficiência simbiótica de fungos previamente selecionados em solo fumigado e quantificar o potencial de inoculação dos fungos no respectivo solo. 


\section{MATE RIAL E MÉTODOS}

O experimento foi realizado em condições de casa de vegetação da E mbrapa Mandioca e Fruticultura, em Cruz das Almas (BA), empregando-se diferentes fungos, exóticos e nativos, considerados eficientes para o mamoeiro em solo fumigado. Utilizou-se amostra de um Latossol o Amarel o álico, col etado na profundidade de 10-30 cm na área experimental da Embrapa, com uso de pastagem nativa. O solo, de textura franco-argilo-arenosa, apresentou 11 esporos deF MA/g de solo; $\mathrm{pH}$ (em água) 4,4; $3 \mathrm{mg} \mathrm{dm}^{-3} \mathrm{deP}$; $31 \mathrm{mg} \mathrm{dm}^{-3} \mathrm{deK}, 8 \mathrm{mmol}_{\mathrm{C}} \mathrm{dm}^{-3} \mathrm{deCa} ; 4 \mathrm{mmol}_{\mathrm{C}} \mathrm{dm}^{-3}$ de $\mathrm{Mg} ; 8 \mathrm{mmol}_{\mathrm{c}} \mathrm{dm}^{-3}$ de Al e $16,1 \mathrm{~g} \mathrm{~kg}^{-1}$ de matéria orgânica. Após passagem em peneira de malha de abertura de $4 \mathrm{~mm}$, parte do solo foi fumigada com bromex $(98 \%$ de brometo de metila e $2 \%$ de cloropicrina), na dosagem de $393 \mathrm{~cm}^{3}$ (lata) por $\mathrm{m}^{3}$ desol o. Em seguida, as duas partes desolo receberam calagem $(1,6 \mathrm{t}$ ha-1) com base no cál cul o do Al trocável $\left(\mathrm{NC}=\mathrm{Al}^{3+} \times 2\right)$, utilizando-se $\mathrm{CaCO}_{3}$ e $\mathrm{MgCO}_{3}$, p.a. em mistura de Ca:Mg de 4:1 (em $\mathrm{mmol}_{\mathrm{c}}^{+}$). Após incubação por sete dias, o pH subiu a 6,3.

O estudo constou de um fatorial $5 \times 8$, com quatro repetições distribuídas em blocos casualizados. Foram testadas cinco doses de $P(0,20,40,80$ e $140 \mathrm{mg} \mathrm{dm}^{-3}$ ) e oito tratamentos fúngicos, referentes a seis tratamentos de inoculação em solo não fumigado, um tratamento com solo não fumigado e sem inoculação (apenas os fungos indígenas) e outro controle, com sol o fumigado, não inoculado, conforme método proposto por Saggin J únior \& Siqueira (1995). O fósforo foi fornecido na forma de superfosfato triplo, apresentando o solo 2, 10, 20, 44 e $85 \mathrm{mg} \mathrm{dm}^{-3}$ de $\mathrm{P}$ (Mehlich-1) para as doses aplicadas, respectivamente. Para corrigir possíveis deficiências de N, K eS, o solo recebeu uma aplicação básica de $80 \mathrm{mg} \mathrm{dm}^{-3}$ de $\mathrm{K}(\mathrm{KCl}), 20 \mathrm{mg} \mathrm{dm}^{-3}$ de $\mathrm{N}$ e $23 \mathrm{mg} \mathrm{dm}^{-3}$ deS $\left[\left(\mathrm{NH}_{4}\right)_{2} \mathrm{SO}_{4}\right]$ misturada no plantio.

Os tratamentos fúngicos constituíram-se de três isolados exóticos [Gigaspora margarita Becker \& Hall, provenientes da Maguari (MG); Acaulospora scrobi culata Trappee G. clarum Nicol son \& Schenck, provenientes da UFLA (MG)] etrês nativos (isolados 22- Glomus sp.; 29- Gigaspora sp. e 35- Acaulospora mellea). Os exóticos foram multiplicados em substrato formado por mistura de solo: areia (3:1), fumigado com bromex, e sorgo granífero (Sorghum bicolor) como planta multiplicadora. Os isolados nativos foram obtidos de plantios de mamoeiro, conforme procedimento descrito na International Collection of Vesicular Arbuscular Mycorrhiza (Invam, 1996), utilizando-se mistura de solo:areia: vermiculita (2:1:1), como substrato, e multi-plicados em sorgo e crotalária (Crotalaria juncea).

Para as inoculações, os fungos foram fornecidos na quantidade aproximada de 500 esporos por planta, sendo inoculados no ato da repicagem, dispondo-seo inóculo (solo, esporos emicorrizas) em torno das raízes da plântula. No tratamento não inoculado em sol o fumigado, fez-se aplicação de um filtrado obtido por passagem de suspensão do solo não fumigado em peneira com malha de abertura de 0,037 um (400 "mesh"). Utilizaram-se plantas de mamoeiro da variedade Tainung no 1 pré-germinadas em mistura de areia + vermiculita (2:1), repicadas quando apresentavam um par defol has definitivas. As plântulas foram transplantadas para os recipientes de cultivo, sacos plásticos com 1,3 dm³ de solo e os inóculos fúngicos.

Dez e 25 dias após a repicagem, fez-se aplicação de solução completa de mi cronutrientes, fornecendo um total de $0,81 \mathrm{mg} \mathrm{dm}^{-3}$ de $B, 3,6 \mathrm{mg} \mathrm{dm}^{-3}$ de $\mathrm{Mn}$, $1,3 \mathrm{mg} \mathrm{dm}^{-3}$ de Cu, 3,9 $\mathrm{mg} \mathrm{dm}^{-3}$ de Zn, 0,15 $\mathrm{mg} \mathrm{dm}^{-3}$ de Mo e 1,6 $\mathrm{mg} \mathrm{dm}^{-3}$ de Fe. Aos 25 dias, fez-se aplicação de $10 \mathrm{mg} \mathrm{dm}^{-3}$ denitrogênio $\left(\mathrm{NH}_{4} \mathrm{NO}_{3}\right)$ via solução nutritiva. O experimento foi concluído 50 dias após a repicagem, quando as plantas foram colhidas para obtenção do peso de matéria seca da parte aérea eteores de nutrientes. Com esses dados, fez-se o cálculo da eficiência de utilização de $P$, conformeSiddiqi \& Glass (1981). Dosistema radicular, tomaram-se amostras para avaliação do percentual de comprimento de raiz colonizada, pelo método da placa reticulada (Ambler \& Young, 1977), após darificação em azul detripano (Phillips \& Hayman, 1970).

Aplicou-seanál ise estatística aos dados e, quando significativa a interação dofungo eP, o efeito do fósforo foi avaliado por meio de regressão com base nas equações ajustadas para matéria seca da parte aérea, conforme as doses deP aplicadas ao solo. Cal cularamse o beneficio de $P$, benefício dos FMAs, eficiência simbiótica e potencial de inoculação, conforme proposto por Saggin J únior \& Siqueira (1995). O benefício do $\mathrm{P}$ foi dado pela área formada entre o eixo X (doses de $\mathrm{P}$ ) e os pontos plotados pela equação de regressão do tratamento não inoculado, em solo fumigado (controle), de acordo com a expressão:

Benefício do $P,(B P) \stackrel{140}{=} \int_{0}^{14} g(x) d x$

O benefício micorrízico foi calculado pela área formada pela diferença entrea curva do tratamento de inoculação ou não, em solo não fumigado, e a do tratamento sem inoculação, em sol o fumigado.

$$
\text { Benefício micorrízico (BM) }=\int_{\mathrm{x} 2}^{\mathrm{x} 1}|f(\mathrm{x})-\mathrm{g}(\mathrm{x})| \mathrm{dx}
$$

em que $f(\mathbf{x})$ é a função ajustada para plantas micorrizadas e $\mathrm{g}(\mathrm{x})$, a função ajustada para plantas não micorrizadas. Os val ores X1 e x2 referem-se ao interval o de doses de P em que a planta micorrizada apresenta maior crescimento quea não micorrizada.

A eficiência simbiótica para cada fungo foi calculada de acordo com a expressão:

$$
\mathrm{ES}(\%)=\frac{\mathrm{BM} \times 100}{\mathrm{BP}}
$$

O potencial de inoculação (PI) decada fungo para o sol oestudado foi cal culado, utilizando-se a seguinte equação: 


$$
\mathrm{PI}(\%)=\left[\frac{\text { Benefício micorrízico do fungo introduzido (exótico ou nativo) }}{\text { Benefício micorrízico dos fungos indígenas (não inoculado, em solo não fumigado) }} \times 100\right]-100
$$

\section{RESULTADOS}

As doses de $\mathrm{P}$ aplicadas ao solo promoveram resposta quadrática no crescimento das plantas e exerceram efeitos na colonização e na resposta destas à inoculação (Figura 1). Observa-se um padrão diferenciado de resposta micorrízica entre os fungos exóticos e os isolados nativos. Para os primeiros, ocorreu grande incremento nas doses mais baixas de $P$, seguindo-se uma tendência de estabilização,
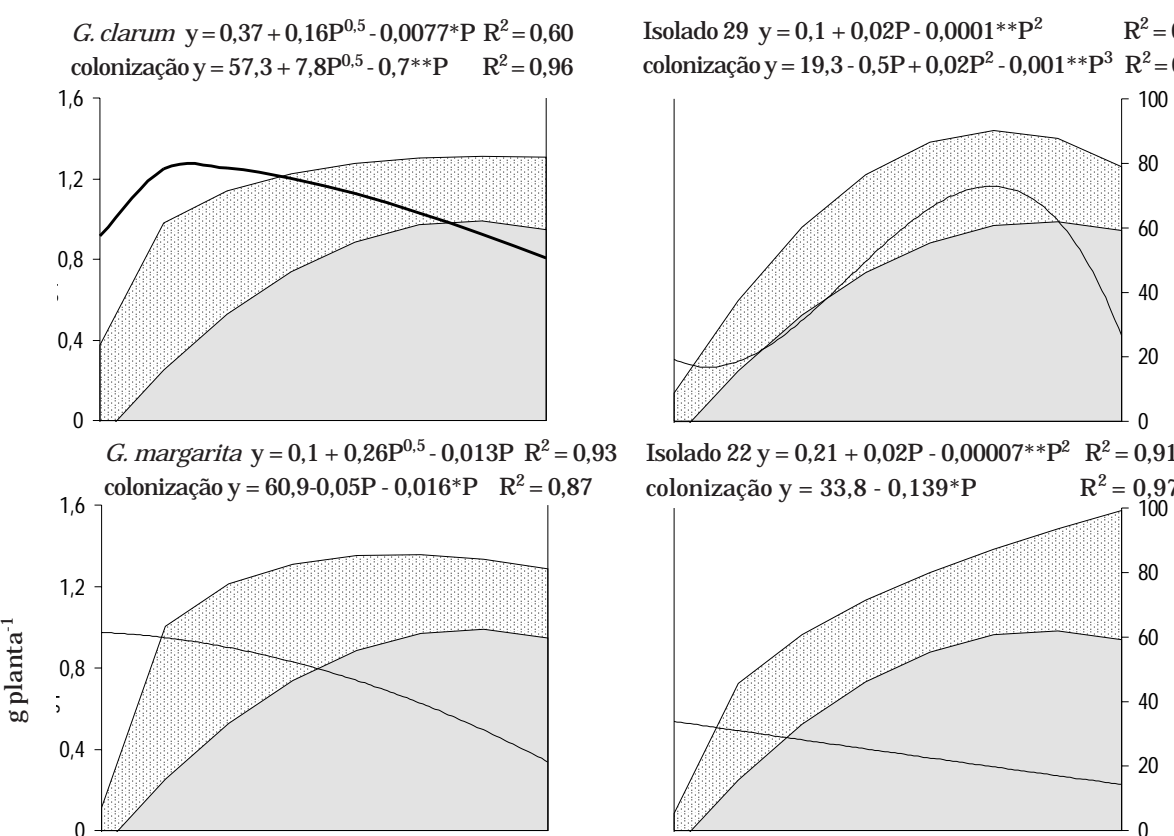

Isolado $22 \mathrm{y}=0,21+0,02 \mathrm{P}-0,00007 * * \mathrm{P}^{2} \mathrm{R}^{2}=0,91$
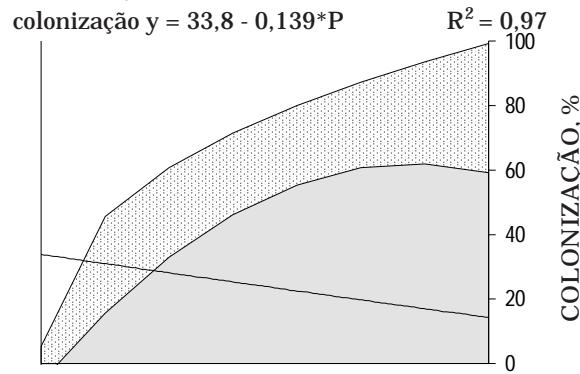

A. scrobiculata $y=0,15+0,23 P^{0,5}-0,0136 * * P R^{2}=0,96$ colonização $y=47,9+5,2 P^{0,5}-0,37 * P \quad R^{2}=0,73$ 1,6
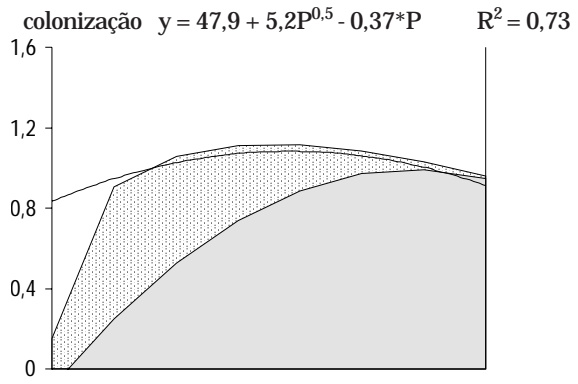

Indígenas $\mathrm{y}=0,08+0,07 \mathrm{P}^{0,5}+0,00043^{*} \mathrm{P} \quad \mathrm{R}^{2}=0,97$ Colonizaçãoy $=29,2+0,48 \mathrm{P}-0,004 * * \mathrm{P}^{2} \quad \mathrm{R}^{2}=0,73$ Controle $y=0,88+0,018 P-0,000079 * * P^{2} R^{2}=0,92$ colonização $y=40,9+1,26 P-0,01 * * P^{2} \quad R^{2}=0,97$
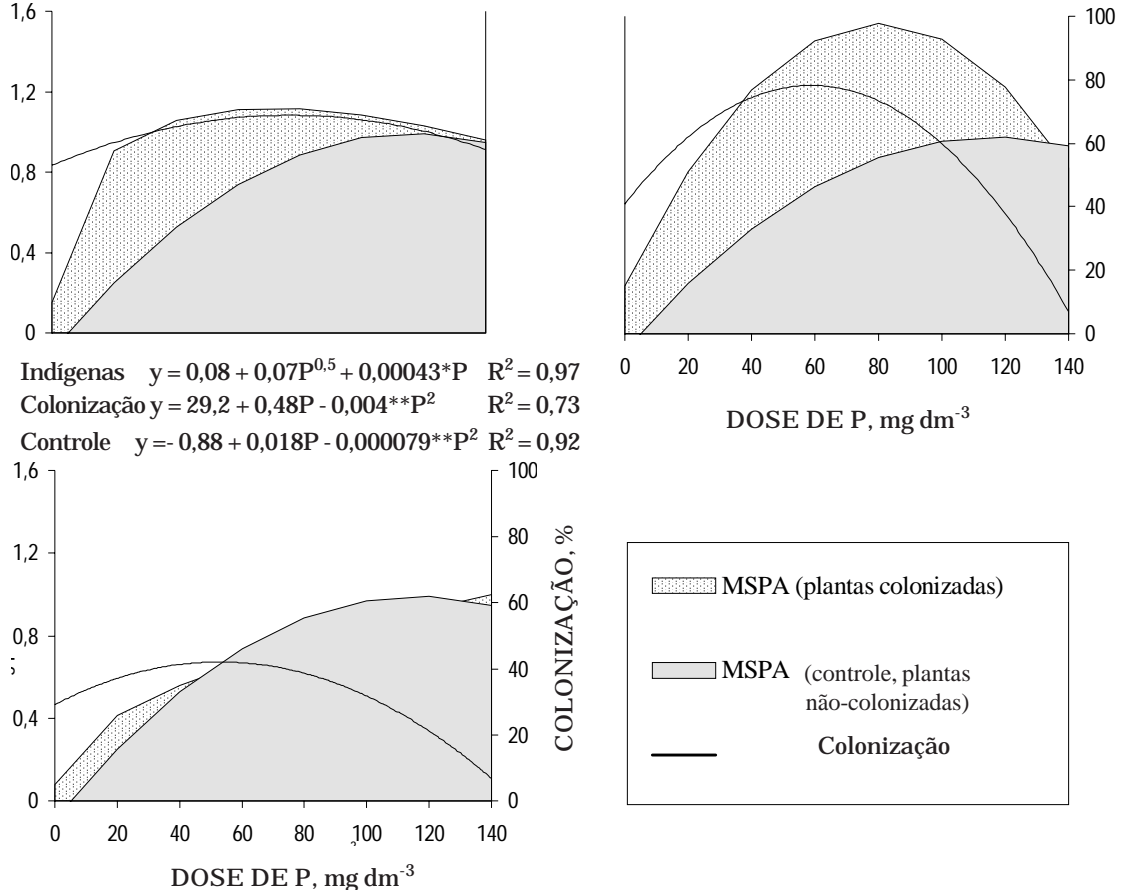

DOSE DE $P, \mathrm{mgdm}^{-3}$

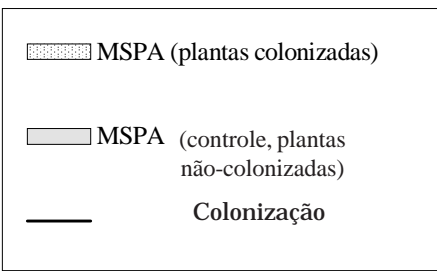

DOSE DE $P, \mathrm{mgdm}^{-3}$

Figura 1. Matéria seca da parte aérea (MSPA) e colonização micorrízica de plantas de mamoeiro, considerando a inoculação de fungos micorrízicos arbusculares em solo não fumigado com doses crescentes de P. (* e** Coeficientes significativos a 5 e 1\%, respectivamente). 
indicada pelo melhor ajuste dos dados ao modelo raiz quadrático. Para os fungos nativos, o modelo quadrático foi o de melhor ajuste, com resposta crescente até doses de $80 \mathrm{mg} \mathrm{dm}^{-3}$ de $\mathrm{P}$, para os isolados 29 e 35 , e até $140 \mathrm{mg} \mathrm{dm}^{-3}$ de $P$, para o isolado 22. Dentre os fungos testados, A. scrobiculata e o isolado 35 causaram crescimento reduzido das plantas na maior dose deP, em relação ao tratamento controle, não colonizado. Entretanto, a máxima produção calculada foi obtida com a inoculação do isolado, referente à dose de $80 \mathrm{mg} \mathrm{dm}^{-3}$ de $\mathrm{P}$. Os fungos indígenas promoveram o crescimento das plantas apenas nas doses mais baixas de $P$, embora o model o ajustado, neste tratamento fúngico, tenha revelado respostas das plantas à aplicação de doses mais el evadas de $P$.

A col onização mi corrízica das raízes de mamoeiro pel os fungos indígenas atingiu valores de até $40 \%$ nas doses intermediárias de $P$, com decréscimo a valores abaixo de $10 \%$ na maior dose deP (Figura 1 ). O padrão de colonização apresentou resposta diferenciada em relação às doses de P. Com exceção do isolado 22 e de G. margarita, que tiveram seu percentual de colonização sempre decrescente com o aumento das doses de $P$, os demais fungos responderam positivamente, em maior ou menor intensidade, aoP. A colonização máxima foi de $80 \%$, para G. clarum; de 67\%, para A. scrobiculata; $71 \%$, para o isolado 29 , e $74 \%$, para o isolado 35 , correspondendo às doses de 22, 78, 98 e $58 \mathrm{mg} \mathrm{dm}^{-3}$ deP, respectivamente. Os val ores de col onização para o isolado 22 foram da mesma ordem dos fungos indígenas. Os fungos G. darum eA. scrobi culata apresentaram valores el evados de colonização, mesmo nas maiores doses deP. Os isolados 29 (Gigaspora sp.) e 35 (A. mel lea) apresentaram el evada sensibilidade à dose mais elevada de $\mathrm{P}\left(140 \mathrm{mg} \mathrm{dm}^{-3}\right)$.

O benefício micorrízico e a eficiência simbiótica dos fungos indígenas foram baixos (Quadro 1). Todos os fungos inoculados apresentaram maior benefício micorrízico que os indígenas, gerando eficiência simbiótica de até $75 \%$, sendo considerada alta para a mai oria dos fungos, com exceção de A. scrobiculata, que apresentou uma eficiência média. A baixa eficiência dos indígenas ea alta eficiência dos fungos inoculados resultaram em elevado potencial de inoculação para todos os fungos, indicando que a inoculação de qualquer um deles pode resultar em benefícios para o mamoeiro, nestas condições. Ressal te-se que, embora a eficiência simbiótica para G. margarita, G. clarum e isolados 22 e 29 tenha sido el evada, estes valores podem estar ainda subestimados, já que as equações de regressão ajustadas (Figura 1 ) revelaram quea inoculação com estes fungos poderia ainda ser benéfica em doses maiores deP.

A aplicação de fósforo ao solo aumentou linearmente os teores desse nutriente nas plantas não col onizadas por fungos micorrízicos (Figura 2). A colonização micorrízica, tanto pelos fungos indígenas como pel os fungos inoculados, aumentou a concentração de fósforo na parteaérea das plantas, nas doses mais baixas de $P$. A inoculação aumentou também a eficiência de utilização do $P$ (Quadro 2), sendo este efeito proporcional mente maior nas doses 20 e $40 \mathrm{mg} \mathrm{dm}^{-3}$ deP. Entretanto, para al guns fungos, a eficiência de utilização foi ainda muito elevada, mesmo na maior dose de $P$, refletindo o grande efeito da inoculação, apresentando os fungos indígenas baixos val ores deeficiência. As plantas nãocol onizadas foram pouco eficientes na utilização do nutriente quando este foi aplicado na dose máxima.

A absorção de potássio também aumentou com a colonização micorrízica, principalmente nas doses mais baixas de $P$ (Quadro 3). Tal efeitofoi semelhante ou até mesmo superior ao efeito provocado pela aplicação de $P$ ao solo. A influência do fósforo na absorção de potássio foi significativa, independentemente da inoculação. A resposta do tipo raiz quadrática para o tratamento controle, não colonizado e fungos indígenas indica um efeito intenso do fósforo do solo sobre a absorção de

Quadro 1. Benefício do fósforo, benefício micorrízico, eficiência simbiótica, categorização e potencial de inoculação de fungos micorrízicos arbusculares para mamoeiro

\begin{tabular}{|c|c|c|c|c|c|}
\hline \multirow{2}{*}{ F ungo } & \multicolumn{2}{|c|}{ Benefício } & \multirow{2}{*}{$\begin{array}{l}\text { Eficiência } \\
\text { simbiótica }\end{array}$} & \multirow{2}{*}{ Categorização(1) } & \multirow{2}{*}{$\begin{array}{l}\text { Potencial de } \\
\text { inoculação }\end{array}$} \\
\hline & Fósforo & Micorrízico & & & \\
\hline & & & $\%$ & & $\%$ \\
\hline Controle & 96,7 & & & & \\
\hline Indígena & & 6,7 & 6,9 & Baixa & \\
\hline G. clarum & & 67,7 & 70,0 & Alta & 914 \\
\hline A. scrobiculata & & 44,5 & 46,0 & Média & 567 \\
\hline G. margarita & & 73,1 & 75,6 & Alta & 996 \\
\hline I solado 22 & & 63,2 & 65,4 & Alta & 848 \\
\hline I solado 29 & & 59,0 & 61,0 & Alta & 784 \\
\hline I solado 35 & & 72,5 & 75,0 & Alta & 987 \\
\hline
\end{tabular}

(1) Segundo Saggin J únior \& Siqueira (1995). 
potássio. Em todas as doses de $\mathrm{P}$, a colonização micorrízica aumentou os teores de Cu na planta com pelo menos um dos fungos, inoculado ou indígena (Quadro 3). Verificou-se, entretanto, comportamento bastante diferenciado entre os fungos: $\mathrm{G}$. clarum eo isolado 22 aumentaram a absorção até $80 \mathrm{mg} \mathrm{dm}^{-3}$ de $\mathrm{P}$, mas Gigaspora margarita efungos indígenas, apenas na ausência de fósforo. $\mathrm{O}$ isolado 29, do gênero
Quadro 2. Eficiência de utilização de fósforo de plantas de mamoiero inoculadas com diferentes fungos micorrízicos, em solo com doses crescentes de $\mathbf{P}$

\begin{tabular}{lccccc}
\hline & \multicolumn{5}{c}{ Dose de P (mg dm } \\
\cline { 2 - 6 } & $\mathbf{0}$ & $\mathbf{2 0}$ & $\mathbf{4 0}$ & $\mathbf{8 0}$ & $\mathbf{1 4 0}$ \\
\hline & \multicolumn{6}{c}{ Eficiência de utilização de P } & $\left(\mathrm{g}^{2}\right.$ & $\left.\mathrm{mg}^{-1}\right)$ \\
Controle & 0,045 & 0,126 & 0,275 & 0,411 & 0,228 \\
Fungos indígenas & 0,062 & 0,187 & 0,153 & 0,249 & 0,389 \\
G. clarum & 0,162 & 0,617 & 0,421 & 0,350 & 0,705 \\
A. scrobiculata & 0,113 & 0,392 & 0,474 & 0,440 & 0,299 \\
G. margarita & 0,080 & 0,400 & 0,651 & 0,564 & 0,503 \\
I solado 22 & 0,072 & 0,407 & 0,557 & 0,600 & 0,961 \\
I solado 29 & 0,073 & 0,317 & 0,490 & 0,657 & 0,557 \\
I solado 35 & 0,097 & 0,513 & 0,533 & 0,689 & 0,286 \\
\hline
\end{tabular}

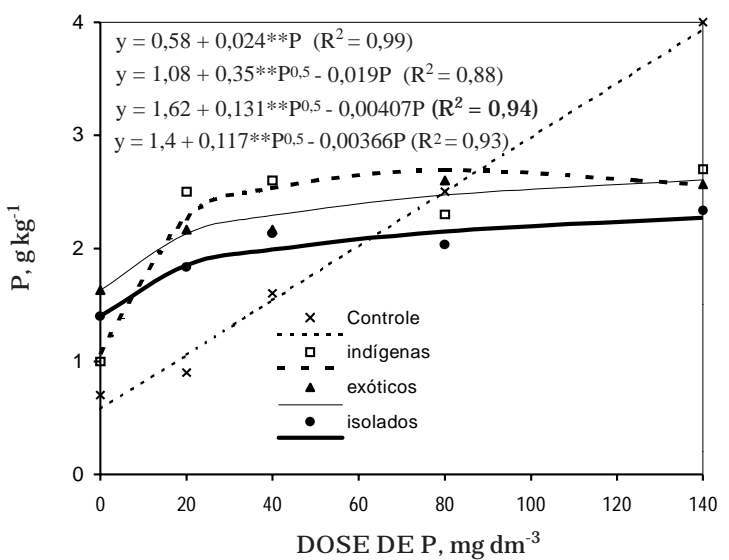

Figura 2. Teores de fósforo na parte aérea de plantas de mamoiero inoculadas com diferentes fungos micorrízicos, exóticos ou isolados nativos, em solo com doses crescentes de P. (** Coeficientes significativos a $1 \%$ ).

Quadro 3. Teores de K, Cu e Zn na parte aérea de plantas de mamoeiro inoculadas com diferentes fungos micorrízicos arbusculares, em solo com doses crescentes de fósforo ${ }^{(1)}$

\begin{tabular}{|c|c|c|c|c|c|c|c|c|}
\hline \multirow{2}{*}{ F ungo } & \multicolumn{5}{|c|}{ Dose de $P\left(\mathrm{mg} \mathrm{dm}^{-3}\right)$} & \multirow{2}{*}{ Regressão } & \multirow{2}{*}{$\mathbf{F}$} & \multirow{2}{*}{$\mathbf{R}^{2}$} \\
\hline & $\mathbf{0}$ & 20 & 40 & 80 & 140 & & & \\
\hline \multicolumn{9}{|c|}{ Potássio (g kg-1) } \\
\hline $\begin{array}{l}\text { Controle } \\
\text { Indígenas } \\
\text { G. clarum } \\
\text { A. scrobiculata } \\
\text { G. Margarita } \\
\text { I solado } 22 \\
\text { I solado } 29 \\
\text { I solado } 35\end{array}$ & $\begin{array}{l}4,3 \mathrm{~d} \\
11,1 \mathrm{c} \\
16,9 \mathrm{ab} \\
18,6 \mathrm{ab} \\
21,2 \mathrm{a} \\
14,0 \mathrm{bc} \\
18,1 \mathrm{ab} \\
16,4 \mathrm{~b}\end{array}$ & $\begin{array}{l}10,5 \mathrm{c} \\
22,5 \mathrm{a} \\
18,6 \mathrm{ab} \\
18,2 \mathrm{ab} \\
19,0 \mathrm{ab} \\
18,6 \mathrm{ab} \\
19,0 \mathrm{ab} \\
17,3 \mathrm{~b}\end{array}$ & $\begin{array}{l}17,7 \mathrm{~b} \\
22,9 \mathrm{a} \\
16,9 \mathrm{~b} \\
17,3 \mathrm{~b} \\
17,3 \mathrm{~b} \\
19,5 \mathrm{ab} \\
20,8 \mathrm{ab} \\
17,3 \mathrm{~b}\end{array}$ & $\begin{array}{l}15,1 \mathrm{~b} \\
16,4 \mathrm{ab} \\
18,6 \mathrm{ab} \\
16,9 \mathrm{ab} \\
20,8 \mathrm{a} \\
19,9 \mathrm{ab} \\
16,0 \mathrm{~b} \\
17,3 \mathrm{ab}\end{array}$ & $\begin{array}{l}14,2 \mathrm{ab} \\
14,7 \mathrm{ab} \\
11,2 \mathrm{~b} \\
13,4 \mathrm{ab} \\
12,5 \mathrm{ab} \\
10,8 \mathrm{~b} \\
10,8 \mathrm{~b} \\
16,9 \mathrm{a}\end{array}$ & $\begin{array}{l}\text { R. quadrática } \\
\text { R. quadrática } \\
\text { Quadrática } \\
\text { Quadrática } \\
\text { Quadrática } \\
\text { Quadrática } \\
\text { Quadrática } \\
\text { Sem ajuste }\end{array}$ & $\begin{array}{l}* * \\
* * \\
* \\
* \\
* \\
* \\
* * \\
\text { ns }\end{array}$ & $\begin{array}{l}0,86 \\
0,84 \\
0,89 \\
0,98 \\
0,68 \\
0,98 \\
0,94\end{array}$ \\
\hline \multicolumn{9}{|c|}{ Cobre (mg kg-1) } \\
\hline $\begin{array}{l}\text { Controle } \\
\text { Indígenas } \\
\text { G. clarum } \\
\text { A. scrobiculata } \\
\text { G. Margarita } \\
\text { I solado } 22 \\
\text { I solado } 29 \\
\text { I solado } 35\end{array}$ & $\begin{array}{c}7,2 \mathrm{c} \\
9,0 \mathrm{bc} \\
9,8 \mathrm{~b} \\
9,4 \mathrm{bc} \\
9,7 \mathrm{~b} \\
13,3 \mathrm{a} \\
9,3 \mathrm{bc} \\
9,2 \mathrm{bc}\end{array}$ & $\begin{array}{c}6,4 \mathrm{c} \\
10,0 \mathrm{a} \\
9,8 \mathrm{ab} \\
10,3 \mathrm{a} \\
7,5 \mathrm{bc} \\
9,3 \mathrm{ab} \\
8,1 \mathrm{abc} \\
8,7 \mathrm{abc}\end{array}$ & $\begin{array}{c}5,7 \mathrm{~d} \\
10,4 \mathrm{bc} \\
11,4 \mathrm{~b} \\
8,1 \mathrm{c} \\
5,8 \mathrm{~d} \\
12,0 \mathrm{~b} \\
16,0 \mathrm{a} \\
9,7 \mathrm{bc}\end{array}$ & $\begin{array}{r}5,9 \mathrm{~b} \\
5,3 \mathrm{~b} \\
9,2 \mathrm{a} \\
5,9 \mathrm{~b} \\
5,5 \mathrm{~b} \\
9,9 \mathrm{a} \\
9,8 \mathrm{a} \\
10,4 \mathrm{a}\end{array}$ & $\begin{array}{l}5,5 \mathrm{~b} \\
7,4 \mathrm{ab} \\
6,6 \mathrm{ab} \\
7,7 \mathrm{ab} \\
5,5 \mathrm{~b} \\
6,1 \mathrm{~b} \\
8,6 \mathrm{a} \\
5,6 \mathrm{~b}\end{array}$ & $\begin{array}{l}\text { R. quadrática } \\
\text { Sem ajuste } \\
\text { Quadrática } \\
\text { Quadrática } \\
\text { Quadrática } \\
\text { Linear } \\
\text { Sem ajuste } \\
\text { Quadrática }\end{array}$ & $\begin{array}{c}* \\
\text { ns } \\
* * \\
* \\
* \\
* * \\
\text { ns } \\
* *\end{array}$ & $\begin{array}{l}0,94 \\
0,86 \\
0,69 \\
0,94 \\
0,72 \\
0,86\end{array}$ \\
\hline \multicolumn{9}{|c|}{ Zinco (mg kg-1) } \\
\hline $\begin{array}{l}\text { Controle } \\
\text { Indígenas } \\
\text { G. clarum } \\
\text { A. scrobiculata } \\
\text { G. Margarita } \\
\text { I solado } 22 \\
\text { I solado } 29 \\
\text { I solado } 35\end{array}$ & $\begin{array}{l}15,9 \mathrm{c} \\
27,8 \mathrm{ab} \\
24,4 \mathrm{abc} \\
28,2 \mathrm{ab} \\
24,2 \mathrm{abc} \\
21,8 \mathrm{bc} \\
34,6 \mathrm{a} \\
23,3 \mathrm{abc}\end{array}$ & $\begin{array}{l}20,2 \text { a } \\
18,5 \text { a } \\
22,8 \text { a } \\
23,8 \text { a } \\
18,5 \text { a } \\
22,0 \text { a } \\
22,7 \text { a } \\
16,1 \text { a }\end{array}$ & $\begin{array}{l}24,0 \text { a } \\
24,6 \text { a } \\
23,0 \text { a } \\
16,9 \text { a } \\
24,0 \text { a } \\
21,3 \text { a } \\
27,1 \text { a } \\
23,3 \text { a }\end{array}$ & $\begin{array}{l}22,4 a b \\
33,0 a \\
21,8 a b \\
13,0 \mathrm{~b} \\
24,6 \mathrm{a} \\
24,2 \mathrm{ab} \\
22,7 \mathrm{ab} \\
22,3 \mathrm{ab}\end{array}$ & $\begin{array}{l}25,3 \text { a } \\
23,1 \text { a } \\
17,6 \text { a } \\
21,8 \text { a } \\
23,1 \text { a } \\
17,8 \text { a } \\
18,3 \text { a } \\
18,7 \text { a }\end{array}$ & $\begin{array}{l}\text { R. quadrática } \\
\text { Sem ajuste } \\
\text { Linear } \\
\text { Quadrática } \\
\text { Sem ajuste } \\
\text { Quadrática } \\
\text { R. quadrática } \\
\text { Sem ajuste }\end{array}$ & $\begin{array}{c}* * \\
\text { ns } \\
* * \\
* * \\
\text { ns } \\
* \\
* * \\
\text { ns }\end{array}$ & $\begin{array}{l}0,90 \\
0,93 \\
0,97 \\
0,73 \\
0,83\end{array}$ \\
\hline
\end{tabular}

(1) Médias seguidas pela mesma letra, na coluna, não diferem significativamente pelo teste de Tukey a $5 \%$. (* e** significativos a 5 e $1 \%$, respectivamente). 
Gigaspora, promoveu maior absorção de $\mathrm{Cu}$ nas maiores doses deP. A absorção de Zn foi influenciada pela presença de fungo micorrízico, tendo sido aumentada apenas na ausência de $\mathrm{P}$ aplicado.

\section{DISCUSSÃO}

Os fungos G. clarum, G. margarita, A. scrobiculata, isolados 22 e 29, eficientes em sol o fumigado, foram também eficientes neste estudo em solo não fumigado, indicando capacidade de competir com os fungos indígenas nas condições utilizadas. Os fungos indígenas mostraram-se pouco eficientes e de baixa infectividade, quando comparados aos introduzidos. I sto pode resultar da baixa densidade de propágulos viáveis e, ou, processo de colonização inicial mais lento, neste caso, decorrente da diluição do inóculo por todoo volume desolo. A eficiência simbiótica obtida e os valores de col onização permitem inferir que os fungos inoculados efetivamente colonizaram as raízes. A localização do inóculo e sua densidade de propágul os podem ter contribuído para esteresultado. Valores de colonização próximos aos apresentados pelos fungos indígenas foram verificados para o isolado 22 (G. etunicatum) e, como este apresentou elevada resposta no mamoeiro, presume-se ser ele capaz de competir com os fungos indígenas. Hepper etal. (1988), verificaramqueumisoladodeG. caledonium, altamente competitivo, foi capaz de excluir os fungos que se achavam disseminados no solo.

O potencial de inoculação indica a previsão de sucesso da inoculação em solo com população indígena. Dessa forma, o el evado valor encontrado para todos os fungos neste estudo, superior ao de trabalho em cafeeiro (Saggin J únior \& Siqueira, 1995), indica que estes fungos podem ser empregados para inoculação do mamoei ro em sol o não fumigado. Dentre os fungos testados, esperava-se maior sucesso com a inoculação de G. margarita, G. clarum e do isolado 35, que apresentaram maiores valores de eficiência (Quadro 1). Entretanto, o isolado 35 mostrou estreita faixa de mutualismo, que pode significar efeitos depressivos em condições de el evadas doses de P. F ungos com el evada col onização como este isolado podem-se tornar parasitas em doses elevadas de fósforo, devido à demanda por carboidratos da planta (Graham et al., 1996). Tal comportamento difere dos obtidos por Saggin J únior \& Siqueira (1995) que encontraram correlação positiva entre o potencial de inoculação e o limite superior da faixa de mutualismo (P aplicado) para os diferentes fungos avaliados, para o cafeeiro, em solo não fumigado. No presente caso, o isolado 35 podeconstituir um fungo sensível a $P$. E $m$ termos práticos, é desejável ter fungos que sejam eficientes em faixa ampla de P disponível no solo.

O comportamento diferenciado dos fungos quanto à el evação nas doses deP indica queos isolados nativos são mais adaptados a condições de maior disponibilidade destes nutrientes, refletindo, possivel mente, as condições de onde foram isol ados, contrariando informações anteriores de trabalhos, como o de J ohnson (1993), segundo o qual fungos isolados de áreas adubadas causaram redução no desenvolvimento de capim andropogon. Essas diferenças ficaram bem exemplificadas na comparação de G. margarita com o isolado 29, pertencenteao mesmo gênero, os quais apresentaram comportamento distinto quanto à colonização e eficiência simbiótica de acordo com as doses de $P$ aplicadas. Diferenças entreisolados da mesma espécie já são documentadas em outros estudos (Medeiros et al., 1994)

Apesar da elevada eficiência simbiótica, mesmo en doses el evadas deP, oisolado 22 apresentou valores de colonização bai xos e decrescentes com a aplicação de P ao solo. I sto pode resultar de uma baixa taxa de disseminação do fungo no sistema radicular (Thomson et al., 1990), afetando o sincronismo entre os organismos ou um efeito direto inibitório do fósforo (Thomson et al., 1991). I sto indica que nem semprea eficiência está relacionada coma col onização, principalmente quando se consideram fungos diferentes, já que a medição da col onização interna total pode não refletir características importantes para o efeito do fungo, como a capacidade de produção de micélio externo (Melloni \& Cardoso, 1999). Para os demais fungos, incluindo os indígenas, a eficiência simbiótica esteve associada a valores el evados de colonização do fungo. Para um mesmo fungo, a colonização diferenciada em função do nível deP no sol otorna-se característica importante da eficiência simbiótica, já que ambas as características são reguladas pel o suprimento de fósforo (Koide, 1991).

A baixa eficiência dos fungos indígenas do solo pode ser explicada por um elevado dreno de carboidratos da planta, uma vez que esses fungos tiveram efeito positivo na absorção de nutrientes ( $P, K, C u$, $\mathrm{Zn}$ ), porém efeito bastante limitado no crescimento da planta. Por sua vez, maior concentração de fósforo na planta nas doses baixas deP nosolo, com aumento concomitanteno crescimento das plantas, indica que G. clarum mostrou grande capacidade de absorção do nutriente e menor custo relativo para a planta, comprovado pelos el evados valores de eficiência de utilização do $P$ pela planta. A maior eficiência de utilização pelas plantas inoculadas pode explicar o mai or crescimento destas, mesmo em doses el evadas deP.

A maior absorção de potássio no mamoei ro como conseqüência da micorrização é consistente com outros estudos com o próprio mamoeiro (Trindade, 1998), podendo ser uma característica desta planta em resposta à colonização pelo FMA. Como a elevação na dose de $P$ elevou os teores de $K$ nas plantas sem micorriza, o efeito dos fungos MA neste nutriente pode ser mediado pelo aumento na absorção de fósforo. Aumento na absorção de K por plantas micorrizadas pode ocorrer mesmo de fontes 
de baixa sol ubilidade (Siqueira \& Ribeiro, 1983). Isto, no entanto, não seaplica a esse estudo, já queo K foi fornecido em forma solúvel. Entretanto, como o K é o nutriente mais absorvido e exportado pelo mamoeiro (Manica, 1982), os F MAs podem estar de fato contribuindo para maior absorção deste elemento, tornando-se mecanismo de grande importância. Como também verificado em outros estudos, a inoculação aumentou a absorção de Cu e Zn (Trindade, 1998). I sto, no entanto, não parece ter relação direta com a eficiência simbiótica desses fungos para o mamoeiro. De fato, os teores desses nutrientes na parte aérea foram pouco influenciados pela el evação na dose de fósforo.

Fungos selecionados para o mamoeiro em solo fumigado foram também eficientes para esta planta, em solo não fumigado, o que também foi verificado em outros trabalhos, com diferentes culturas (Manjunath et al., 1983; Saggin J únior \& Siqueira, 1994; Daniels-Hylton \& Ahmad, 1994), demonstrando a possibilidade de obter efeitos da inoculação nestas circunstâncias. Estes fungos devem ser testados em campo, onde a competição dar-se-á também ao longo do tempo.

\section{CONCLUSÕES}

1. Todos os fungos testados apresentaram eficiência simbiótica para o mamoeiroTainung no 1 em solo não fumigado, destacando-se G. clarum, G. margarita e isolado 29 (Gigaspora sp), que são promissores para programas de inoculação de mudas.

2. O desenvolvimento do mamoeiro, no solo em estudo, poderá ser maximizado pela introdução de fungos micorrízicos e uso de menores doses de fósforo.

3. Os isolados nativos foram mais eficientes em aumentar a produção de parteaérea do queos exóticos, em doses mais elevadas de fósforo no sol o.

4. A eficiência simbiótica relacionou-secom uma maior absorção e eficiência de uso de fósforo e maior absorção de potássio pelo mamoeiro.

\section{LITERATURA CITADA}

AMBLER, J.R. \& YOUNG, J .L. Techniques for determining root length infected by vesicular-arbuscular mycorrhizae. Soil Sci.Soc.Am. J ., 4:551-556, 1977.

AULER, P.A.M. Desenvolvimento inicial do mamoeiro (Carica papaya L.) relacionado à disponibilidade de fósforo no solo e à colonização pelo fungo micorrízico vesículo-arbuscular Glomus macrocarpum. Botucatu, Universidade Estadual de São Paulo, 1995. 94p (Tese de Mestrado)

BALAKRISHNA, R.; BAGYARAJ, D.J .; MALLESHA, B.C. \& REDDY, B. Selection of efficient VA mycorrhizal fungi for papaya. Biol. Agric. Hort., 13:1-6, 1996.
DANIELS-HYLTON, K.D.M. \& AHMAD, M.H. Inoculation response in kidney beans (Phaseolus vulgaris, L.) to vesicular-arbuscular mycorrhizal fungi and rhizobia in nonsterilized soil. Biol. Fert. Soils, 18:95-98, 1994.

GRAHAM, J .H.; DROUILLARD, D.L. \& HODGE, N.C. Carbon economy of sour orange in response to different Glomus spp. Tree Physiol., 16:1023-1029, 1996.

HEPPER, C.M.; AZCON-AGUILAR, C.; ROSENDAHL, S. \& SEN, R. Competition between three species of Glomus used as spatially separated introduced and indigenous mycorrhizal inocula for leek (Allium porrum L.). New Phytol., 110:207215, 1988.

INTERNATIONAL CULTURE COLLECTION OF ARBUSCULAR E VESICULAR MYCORRHIZAL FUNGI - INVAM. Disponível: Site I nvam (08 de jun. 1996). URL: http://invam.caf.wvu.edu/myc_info/methods/cultures/ monosp.htm Consultado em 12 fev., 1997.

J OHNSON, N.C. Can fertilization of soil select less mutualistic mycorrhizae? Ecol. Applic., 3:749-757, 1993.

KOIDE, R.T. Nutrient supply, nutrient demand and plant response to mycorrhizal infection. New Phytol., 117:36586, 1991.

MANICA, I. Fruticultura Tropical: 3. Mamão. São Paulo, Ceres, 1982. 255p.

MANJ UNATH, A.; MOHAN, R. \& BAGYARAJ , D.J . Response of citrus to vesicular-arbuscular mycorrhizal inoculation in unsterile soils. Can. J. Bot., 61:2729-2732, 1983.

MEDEIROS, C.A.B.; CLARK, R.B. \& ELLIS, J .R. Growth and nutrient uptake of sorghum cultivated with vesiculararbuscular mycorrhiza isolates at varying pH. Mycorrhiza, 4:185-191, 1994.

MEDINA, O.A.; SYLVIA, D.M. \& KRETSCHMER J r., A.E. Response of siratro to vesicular-arbuscular mycorrhizal fungi: II. Efficacy of selected vesicular-arbuscular mycorrhizal fungi at different phosphorus levels. Soil Sci. Soc. Am. J ., 52:420-423, 1988.

MELLONI, R.\& CARDOSO, E.J.B.N. Quantificação de micélio extrarradicular de fungos micorrízicos arbusculares em plantas cítricas. I. Método empregado. R. Bras. Ci. Solo, 23:53-58, 1999.

MOHANDAS, S. Effect of VAM inoculation on plant growth, nutrient level and root phosphatase activity in papaya (Carica papaya cv. Coorg Honey Dew). Fert. Res., 31:263267, 1992.

PHILLIPS, J .M. \& HAYMAN, D.S. Improved procedures for clearing roots and staining parasitic and vesiculararbuscular mycorrhizal fungi for rapid assessment of infection. Trans. Br. Mycol. Soc., 55:158-161, 1970.

POWELL, C.L.; GROTES, M. \& METCALFE, D. Mycorrhizal inoculation of barley crop in the fields. N.Z.J . Agric. Res,. 23:107-109.1980.

RAMIREZ, B.N.; MITCHELL, D.J. \& SCHENCK, N.C. Establishment and growth effects of three vesiculararbuscular mycorrhizal fungi on papaya. Mycologia, 67:1039-1041,1975.

SAGGIN J ÚNIOR, O.J . \& SIQUEIRA, J .O. Interação fungos micorrízicos versus superfosfato e seus efeitos no crescimento e teores de nutrientes do cafeei ro em solo não fumigado. R. Bras. Ci. Solo, 18:27-36, 1994. 
SAGGIN J ÚNIOR, O.J \& SIQUEIRA,J .O. Avaliação da eficiência simbiótica de fungos endomicorrízicos para o cafeeiro. R. Bras. Ci. Solo., 19:221-228, 1995.

SIDDIQI, M.Y. \& GLASS, A.D.M. Utilization index: a modified approach to the estimation and comparison of nutrient utilization efficiency in plants. J . Plant Nutr., 4:289-302, 1981.

SILVA, L.F.C. \& SIQUEIRA, J .O. Crescimento e teores de nutrientes de mudas de abacateiro, mangueira e mamoeiro sob influência de diferentes espécies de fungos micorrízicos vesículo-arbusculares. R. Bras. Ci. Solo, 15:283-288, 1991

SIQUEIRA, J .O. Fisiologia e bioquímica de micorrizas vesículoarbusculares: alguns aspectos da relação fungo-planta e absorção de fósforo. In: REUNIÃO BRASILEIRA SOBRE MICORRIZAS, 4., Mendes, 1991. Programa e Resumos. I taguaí, EMBRAPA-CNPMBS/UFRRJ , 1991. p.105-131.

SIQUEIRA, J.O. \& RIBEIRO, M.A.V. Efeito da micorriza vesicular-arbuscular (MVA) na produção de matéria seca e absorção de potássio do sienito nefelínico de Poços de Caldas pelo milho e soja. Ci. Prát., 7:197-204, 1983.
THOMSON, B.D.; ROBSON, A.D. \& ABBOTT, L.K. Mycorrhizas formed by Gigaspora calospora and Glomus fasciculatum on subterranean clover in relation to carbohydrate concentration in roots. New Phytol., 114:217-225, 1990

THOMSON, B.D.; ROBSON, A.D. \& ABBOTT, L.K. Soil mediated effects of phosphorus supply in theformation of mycorrhizas by Scutellospora calospora (Nicol. \& Gerd.) Walker and Sanders on subterranean clover. New Phytol., 118:463-469, 1991

TRI NDADE, A.V. Micorrizas arbusculares em mamoeiro. Lavras, UFLA, 1998. 178p (Tese de Doutorado)

WEBER, O.B. \& AMORIM, S.M.C. Adubação fosfática e inoculação de fungos micorrízicos vesículo-arbusculares em mamoeiro “Solo". R. Bras. Ci. Solo, 18:187-191, 1994.

WILSON, J .M. Competition for infection between vesiculararbuscular mycorrhizal fungi. New Phytol., 97:427-435, 1984. 
A. V. TRINDADE et al. 